\title{
Development of Physiological Parameters Monitoring System using the Internet of Things
}

\author{
https://doi.org/10.3991/ijoe.v13i09.7436 \\ Haoran Li, Tianhong Pan $\left({ }^{\varpi}\right)$ \\ Jiangsu University, Zhenjiang, China \\ thpan@ujs.edu.cn
}

\begin{abstract}
To improve people's quality of life, a remote healthcare service with Internet of things (IoTs) is developed in this paper. The proposed system includes microcomputer, physiological sensors and corresponding signal preprocessing circuit, and wireless transmitting module etc. Sensors are used to detect the various vital signals. The collected data is transmitted to mobile device using Bluetooth. The doctors get patient's physiological parameters through the GPRS which connects the mobile device and medical center. As a result, doctor can give diagnostic message to the patient without face to face. The detecting device is designed as a watch form which is wrist-worn enclosure, unobtrusive and low power consumption. An android mobile app is also designed to transmit data as well as display the collected data. The experiment validates the performance of the proposed system.
\end{abstract}

Kewords-Internet of things, Physiological parameters, Bluetooth, GPRS

\section{$1 \quad$ Introduction}

The lack of medical resource (such as the shortage of doctors and hospitals) has being become an important social issue due to population increasing and ageing. The traditional medical diagnosis mode (face to face) cannot satisfy people' requirements [1]. In order to solve this issue, the concept of telemedicine is proposed. Using the telemedicine, the medical system runs more efficiently than before, especially monitors the patients with the chronic diseases. Recently, more and more advanced technologies and conceptions are proposed. For healthcare, the internet of things (IoTs) and the wearable devices promote the development of telemedicine [2]. IoTs is a good medium in telemedicine, which integrates equipment and expertise in the medical field together [3]. The wearable device combines the sensors and processing devices into a single device, which is suitable for monitor. The patient can take wearable device everywhere without any limitation.

Mobile monitoring of physiological parameters has been studied by many research groups and some physiological parameters collection system have been developed for healthcare care, such as data acquisition (e.g. for ECG) [5], personal monitors [6] and so on. Researchers in University of Alabama have designed a wearable ECG monitor with real time feedback to the user [7]. Sensors can be integrated by using a wireless 
body area network [8]. However, there are still some sensors that are not integrated into a single device. In the medical equipment market, there are several products in sales. Manufacturers (such as Agilent, Phillips and Nellcor) produce handheld pulse oximeters for non-invasive monitoring of blood oxygen saturation and pulse (SpO2). Omron designs a range of portable wrist devices for measuring blood pressure. The chest-worn polar measures heart rate, but doesn't provides further information about ECG, QT or QRS [9]. Furthermore, the chest-worn polar also let users feel uncomfortable. Most of these devices only measure a single parameter, and then the collect system needs several devices. In addition, these devices don't provide the monitoring function.

A smart mobile is becoming a reliable personal communications channel, and the electronic communications become reliable, secure and convenient [10]. For the smart phone, message function, data shortage and application are the basic functions, which let it be an excellent transfer platform.

In this paper, a physiological monitoring system with IoTs was proposed. The proposed system comprises three separate parts: physiological parameters collection module, mobile device and remote monitoring unit (shown in Fig.1.).

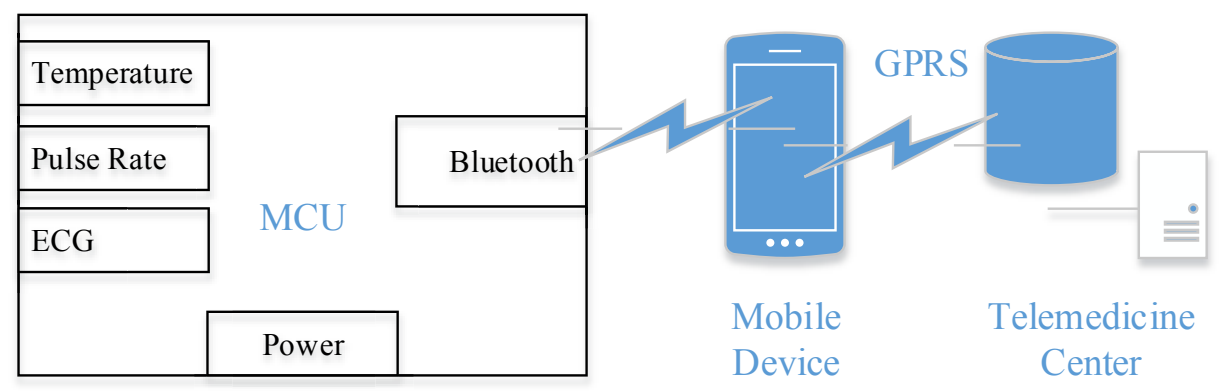

Fig. 1. The header image of online-journals.org

Sensors, MCU and signal processing circuits were integrated in a single wrist device. The patient could wear this device everywhere without any limitation. Various sensors and microprocessor were used to collect the physiological parameters (ECG, heart rate and body temperature). The collected parameters were transferred to mobile device (with android platform) using Bluetooth. The user could get their physiological parameters through monitor application. At last, the GPRS was used to transmit the collected data from the mobile device to the telemedicine center. The doctor who works in the telemedicine center can use the collected data to make diagnosis.

The rest of the paper is organized as follows. Section 2 describes the design of wearable device. Section 3 gives the App development. Section 4 shows the testing results. Finally, section 5 summarizes the development and presents the future works. 


\section{The Parameter Collection Modules}

The parameter collection module is divided into six parts: MCU, power supply, heart-rate collection, body temperature collection, ECG collection and Bluetooth. The principle of components selection is based on performance, power consumption and physical size.

\subsection{MCU}

A CC2540 with low power consumption was used as the MCU, which is responsible for collecting, buffering, and transferring data from related interfaces. The chip combines an excellent RF transceiver with an industry-standard enhanced 8051MCU, in-system programmable flash memory, 8-KB RAM, and many other powerful features and peripherals. In addition, a 12-bit ADC is also integrated in the CC2540. In different working modes, the power consumption of CC2540 varies from $0.0005 \mathrm{~mA}$ $18.2 \mathrm{~mA}$

\subsection{Power-supply}

It is well known that the portable equipment should have small size and less weight. Furthermore, the power consumption should be low or batteries should be charged easily. In order to meet these requirements, button cell battery was selected as the power supply. But the voltage of temperature sensor and heartbeat sensor are $3.3 \mathrm{~V}$. Here, a linear regulator AMS1117-3.3 is used to transform the voltage into 3.3V (shown in Fig.2). A magnetic reed switch MK24-B-2-OE is placed between the batteries and the inputs of the power regulators, so the system power can be switched on or off from outside.

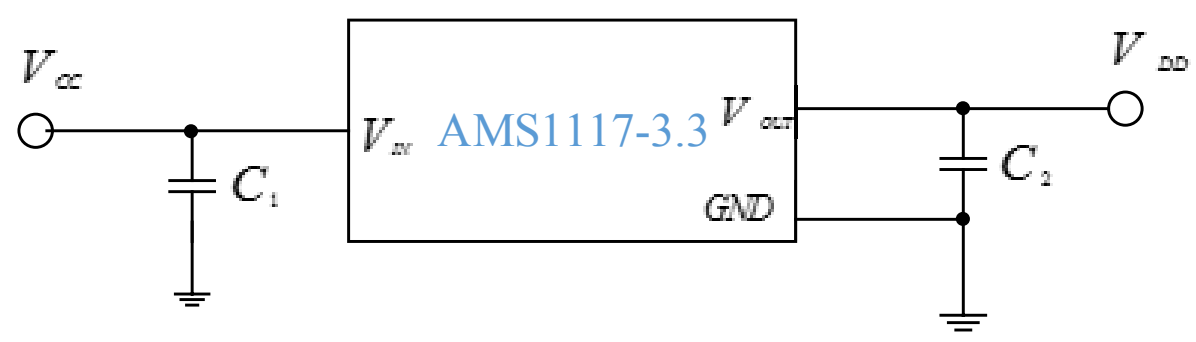

Fig. 2. DC-DC circuit

\subsection{Heart-rate collection}

Heart-rate is a very important indicator for both amount of exercise and motion function [11].There are three main methods for the heart-rate collection.

1. Collect ECG and pulse signal.

2. Use pressure sensor to collect the fluctuation and calculate the pulse rate. 
3. Photo elective volume method [12].

The method 1 and 2 have limitation on patients' activities and let patient fell uncomfortable. The last one is widely used in heart-rate measurement. Furthermore, the small size is suitable for this system.

The vital signal are weak, but the environment is full of noise. So the vital signals are easy to be affected. As a result, the collected physiological parameters are inaccuracy which will mislead the diagnosis. In order to eliminate the noise of the measurement, the corresponding signal processing circuit including two stage amplifiers are designed (shown in Fig.3). AD620 amplifier is chosen as the main component in first stage. In order to prevent signal distortion, the gain is set at $10 \mathrm{db}$. The amplifier in the second stage is to make the voltage signal be suitable for the requirement of $A / D$ converter.

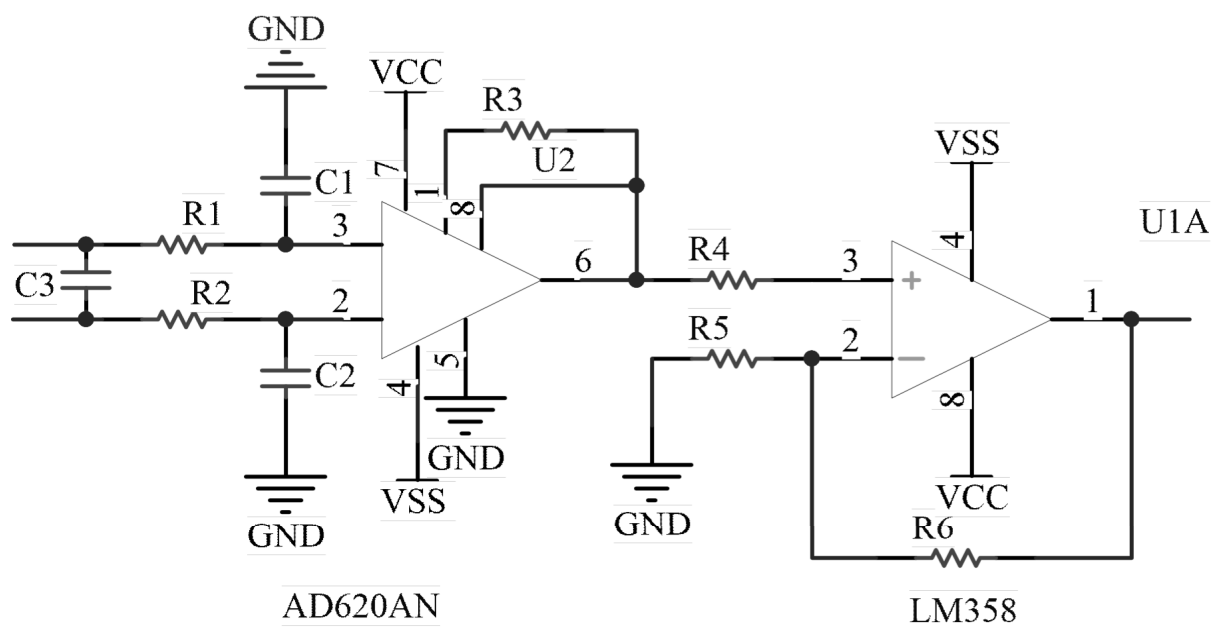

Fig. 3. The schematic of amplifier

\subsection{Body temperature collection}

The body temperature is the most important physiological parameter for human health. The clinical experiments show a fact that the increase of body temperature is the warning signal of some diseases. It is necessary to monitor the body temperature, especially for the patient. In the developed device, the DS18B20 is selected as the temperature sensor, which only has 1 -wire interface. The collected data can be converted to 12 -bit digital word directly. The range of DS18B20 is $\left[-55^{\circ} \mathrm{C},+125^{\circ} \mathrm{C}\right]$. In addition, the sensor has feature of anti-interference and low-cost.

\subsection{ECG collection}

The heart muscle consists of a group of fusion cells. The electrical signals can be used to draw an Electrocardiogram (ECG) which is an indispensable tool for diagnos- 
ing heart disease. To obtain the ECG, the patient must be physically connected to a front-end amplifier with special bio-electrodes. Bio-electrodes convert ionic current flow to the electronic flow. The material of bio-electrodes is important for this part, because the metal will be oxidized. In this design, the golden bio-electrodes are selected [13]. The frequency of ECG signal is $0.05 \mathrm{~Hz} \sim 100 \mathrm{~Hz}$. In order to obtain ECG signal with high accuracy, two main noises should be filtered (i.e. baseline shifting, electromagnetic field interference with high frequency). So, a high pass filter (cut off is $0.5 \mathrm{~Hz}$ ) and a low pass filter (cut off is $100 \mathrm{~Hz}$ ) are integrated in the amplifier stages.

\subsection{Data transmission}

The Bluetooth 4.0 technology is selected as the communication media between collection system and mobile device, because of convenience. The Bluetooth in collection system is based on CC2540. And the Bluetooth Low Energy (BLE) was used in this part. The BLE protocol stack is built from physical layer in the bottom to application layer in the top. Any profiles and applications that are used on top of the GAP and GATT layers of the stack. GAP defines how to discover and build a connection with other device and other general access function. GATT describes a use case, roles and general behaviors based on the GATT functionality

The GPRS was used as a bridge between mobile device and telemedicine center. The collected parameter in the mobile phone can be transferred to server in the telemedicine center directly.

\section{APPs Development}

In this system, the APPs was designed by using the Java Development kit (JDK) 7, Android SDK platform and Eclipse open-source Java development program. There are four steps in the development of this apps

1. Analysis: this step including the knowledge requirement, functional requirement and algorithm design

2. Apps Design: the researcher or programmers designed the app based on the need of patient and professionals.

3. Testing the prototype: the patient and professionals should use the App and give some suggestions. Besides this, professionals should develop the content of this Apps (such as health knowledge and videos).

4. App development: programmer develop the software based on the suggestions. The process of App design is shown in Fig.4. 


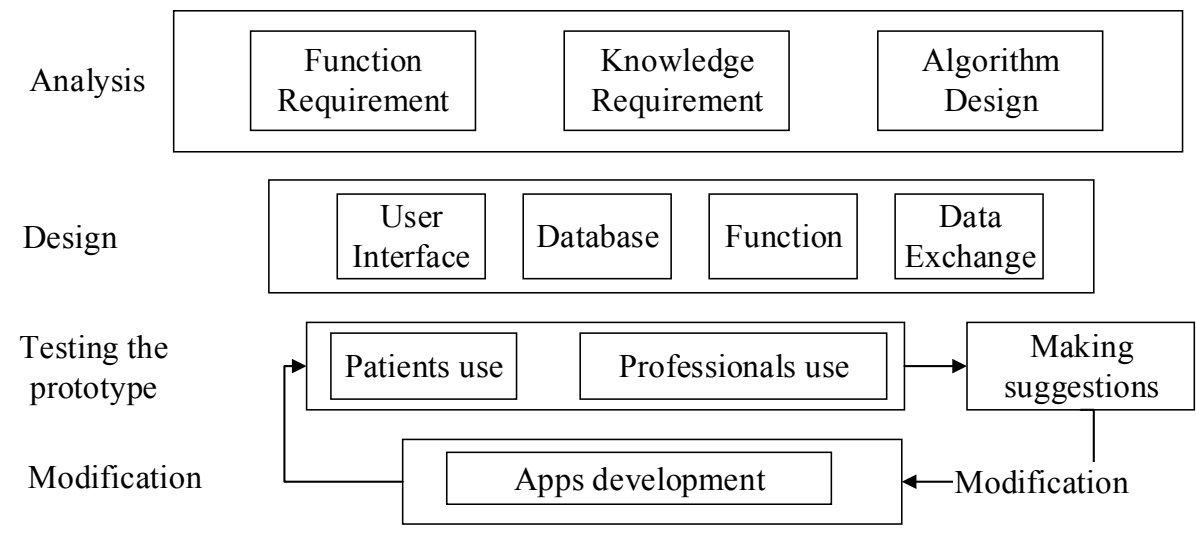

Fig. 4. Procedure of healthcare application design

\subsection{UI design}

Through various navigation options (such as parameter display, health knowledge), the user can browse the information, such as their physiological parameter and health knowledge. The UI of the proposed healthcare application is shown in Fig.5. The layout and interface of our application were designed with the conception of "easy to use". The patients' personal information and physiological parameters should be kept secretly. First of all, a login interface of UI is required, and patients can build up their own account and password. In the modification stage, the heuristics evaluation is used [14]. In this work, a heuristic checklist is used for accessible smartphone interface design, developed through reviewing existing design standards and guidelines. 


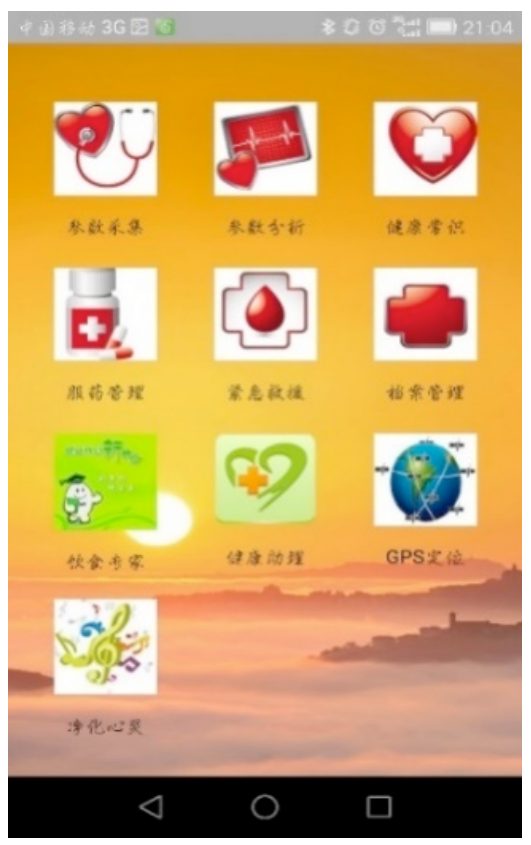

Fig. 5. User interface of Healthcare application

\subsection{Data transmission}

First, the Android application should search for the Bluetooth device which is in the collection device. And then, the connection is established. Next, the data receiving is start. Using 'Handler' to realize the asynchronous processing. The received data will be stored in SQLite database and JavaBean file. In addition, A FLASH chip is carried on the MCU. The data can be stored in the FLASH and it will be sent automatically when the communication between devices is established. AT45DB161D$\mathrm{SU}$ is selected as data flash, its storage capacity is $16 \mathrm{M}$, and the supply voltage range is $2.7 \mathrm{~V}-3.6 \mathrm{~V}$, the maximum operating current is $15 \mathrm{~mA}$.

\subsection{Data storage and query}

All the physiology parameters not only stored in SQLite database but also stored in SD card. First, '/sdcard/' is used to create a File object, then, "getAbsolutePath ()' is set to achieve the Storage path. In this case, the storage handler will run simultaneously on background to save raw data.

File dir. =new File ("/sdcard/");

String SDPath=dir.getAbsolutePath ();

Using 'CreatNewFile' to create a file. At least, using the 'write ()' to write the message in the file. The user can click the "save" to storage their physiology into the SD card.

The Operation effect of data receive and storage is shown in Fig.6. 

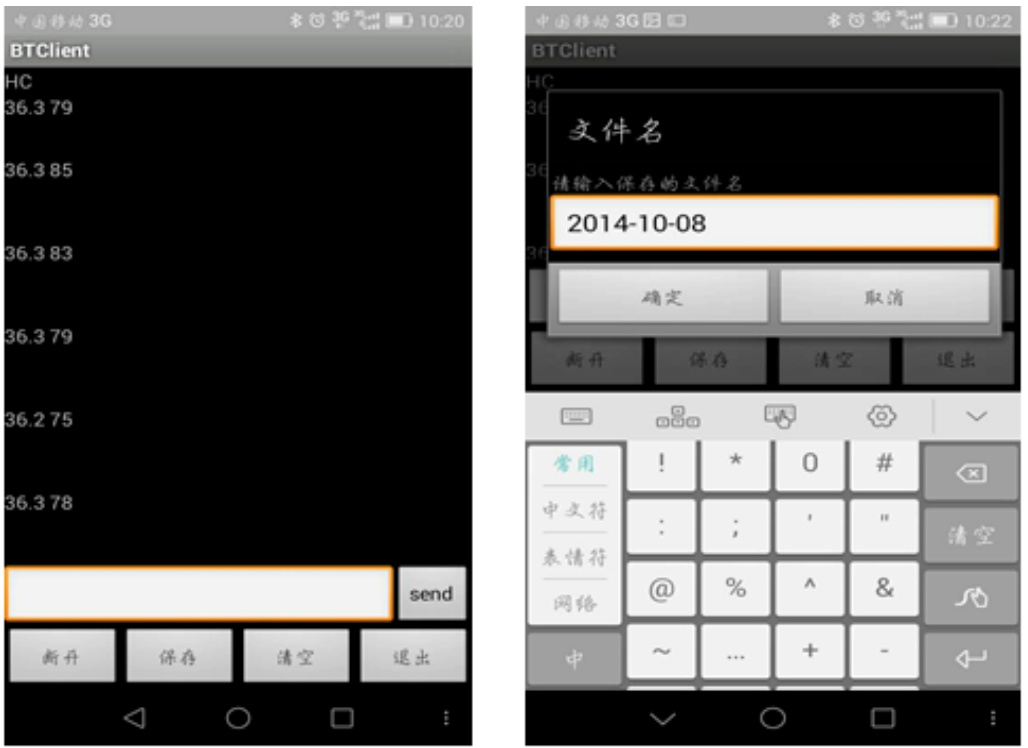

Fig. 6. Operation effect of data receive and storage

\subsection{Historical data query}

The patient can search the historical data by query function. In order to realize query function by times, sqlFan=" $1=1$ " was defined to feedback all data in the database. Array 'date []'was used to shortage the start time and end time. (The Operation effect of history parameter query was shown in Figure.7)

The code of quarry function:

/* 'Ftime' is a start time, 'Ttime' is an end time */

if(!Ttime.trim().equals(" ")\&\&! Ftime.trim().equals(" "))

showToastMsg("According to the start and end time! "); sqlFan_+=" and time_ $>$ ? and time_< ? ";

date $=$ new String $[2]$;

date [0]=Dateutil . parseStr2Str (Ftime,

DateUtil.YYYY_MM_DD_HH_MM);

date [1] =Dateutil.parsestr2Str $($ Ttime,

DateUtil.YYYY_MM_DD_HH_MM);

\}

/**search for user information, display by list */ DataBeanList

DbTools.queryData (SearchDataActivity.this,

sqlFan, date);

if (list! =null) \{

bundel.putSerializable("searchList", list);

jumpToPage (SeachResultActivity.class, bundel, false);

\} 


\subsection{Message function}

The application was designed with the permission for reading text message. The message is from telemedicine center (such as doctor's diagnosis). In addition, warning function is designed to draw user's attention. If the value of collected parameters were beyond the normal value, a warning information will appear in the notification bar. (Shown in Fig.8).

The code of warning function:
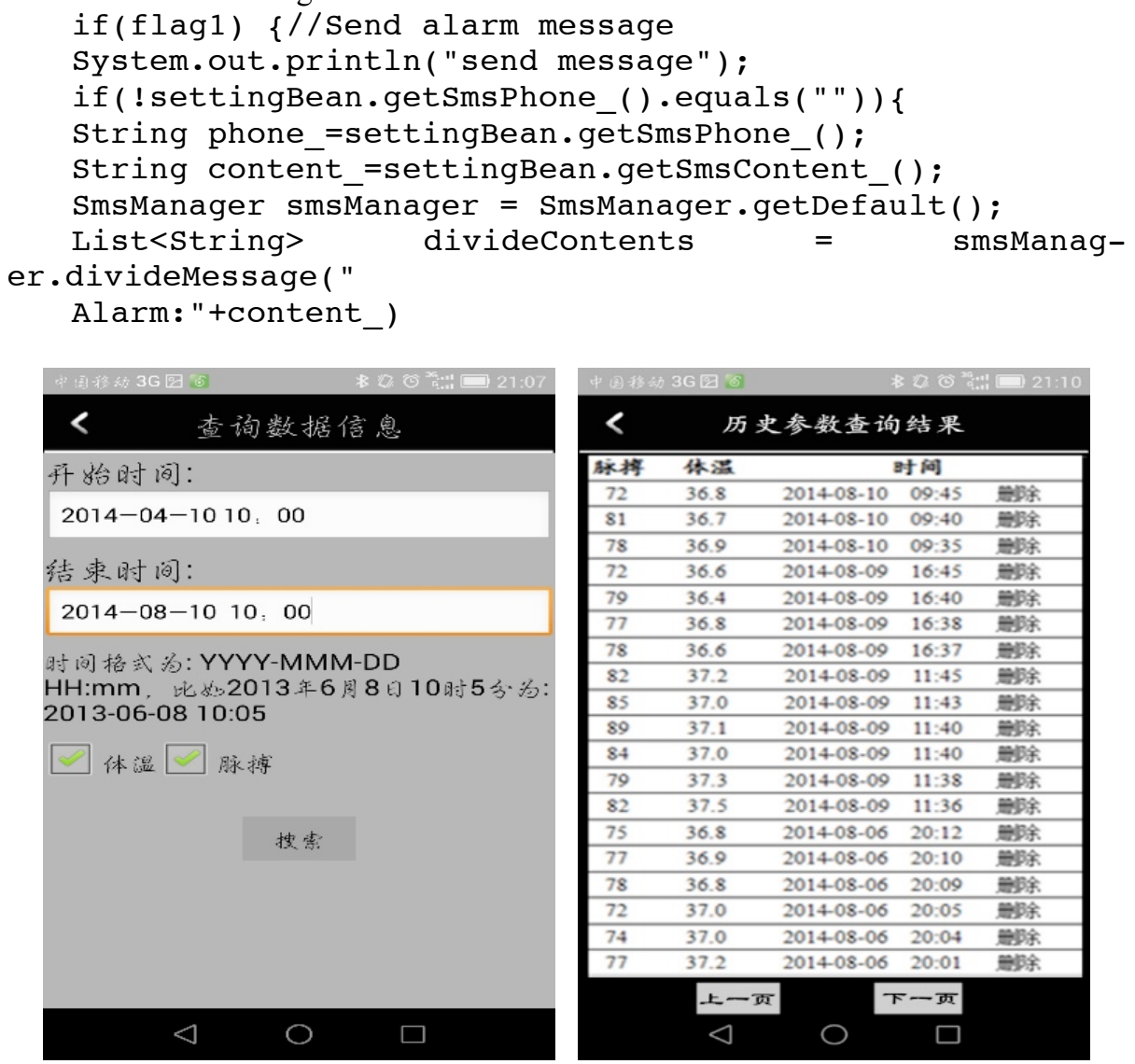

Fig. 7. Historical data query 


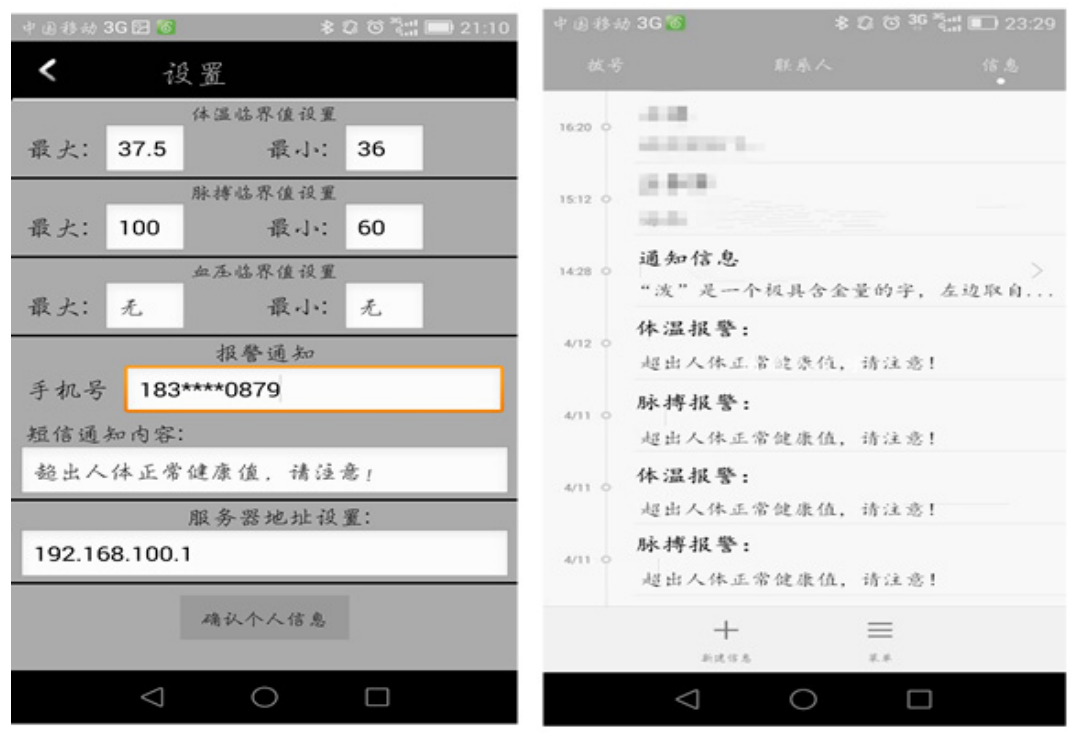

Fig. 8. Alarm function

\section{System Test}

The test focuses on accuracy of measurement and system's power consumptions.

\subsection{Parameter collection test}

In this paper, a remote monitoring healthcare system has been designed for medical institutions to meet public's requirements, especially for patients who need all-time monitor or live lonely. In addition, doctors can monitor different patients simultaneously through the system, which can improve the efficiency of monitor. The performance of the system has been validated by a series of experiments. The parameter collection results and EGC collection function is shown in Fig. 9. 


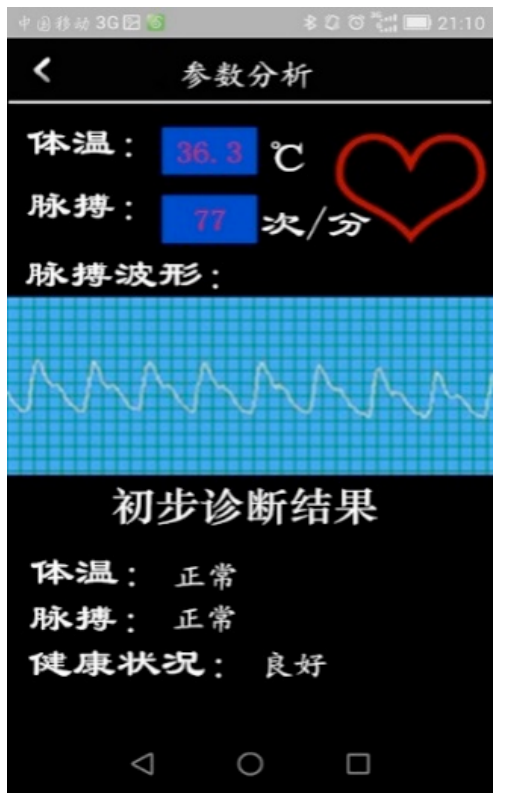

Fig. 9. The parameter collection results

\subsection{Accuracy detection}

The accuracy of collected parameters directly affects the doctor's diagnosis. In order to test the accuracy, the professional equipment was taken for comparison. For heart rate measurement, Classic-70 multi-parameter monitor produced by Health force was used, HW-5 thermometer was used to measure body temperature. As shown in Table 1, the accuracy of the developed system was high enough.

Table 1. Comparison between two devices

\begin{tabular}{|l|c|c|c|c|}
\hline \multicolumn{1}{|c|}{ Items } & System Collect(AVG) & Equipment collect(AVG) & Error & Relative error \\
\hline Heart rate & 77.4 & 77 & 0.4 & $0.52 \%$ \\
\hline Body Temperature & 36.49 & 36.41 & 0.08 & $0.22 \%$ \\
\hline
\end{tabular}

\subsection{Power consumption test}

Low power-consumption is one of the major targets in this systems. The power consumption of Bluetooth module in two different condition (Working state and Sleep state) were tested and shown in Table 2. It can be seen that the working current was less than $24 \mathrm{~mA}$, which was good enough for this system.

Table 2. Power consumption analysis

\begin{tabular}{|l|l|l|l|l|l|l|}
\hline \multicolumn{1}{|c|}{ Item } & No.1 & No.2 & No.3 & No.4 & No.5 & No.6 \\
\hline W Working Current(mA) & 15.11 & 15.09 & 15.13 & 15.15 & 15.09 & 15.10 \\
\hline Sleep Sleeping Current(mA) & 0.004 & 0.005 & 0.004 & 0.004 & 0.005 & 0.004 \\
\hline
\end{tabular}




\subsection{Discussion}

Compared with existing devices, the system has several unique features as follows.

1. A smart phone with android platform is used as data logger which eliminates the need to carry a bulky conventional data recorder.

2. This system is capable to measuring body temperature, heartbeat and ECG.

3. The main communication methods are Bluetooth and GPRS, which means that the system does not need build an extra communication network.

4. The collected parameters will be sent to telemedicine center by GPRS. At the telemedicine center, medical data from mobile device is collected and processed by doctors. If there are something wrong in patients' parameters, the alarm message will be feedback to the mobile device by GPRS.

\section{Conclusion}

A physiological parameters monitoring system based on the internet of things is proposed in this paper. The system combines multi-parameter measurement of vital signals, smart mobile device, online analysis and emergency detection. All of the sensors and microprocessor are integrated in a single device. Android smart phone play a role of bridge between the patient and telemedicine center.

There are several works in the future. One of them is to develop the telemedicine center for viewing and diagnosis. The other is the location function. The potential technology is the global positioning system (GPS) which locates positions anywhere on the Earth. Another solution is to select the Wi-Fi which offers wireless internet service at millions of hotspots. This widespread infrastructure offers the possibility to locate mobile devices in an economical way. Position determination using Wi-Fi technology has the advantage that it can perform indoors and outdoors.

\section{Acknowledgment}

The authors would like to thank the financial support provide by the National Natural Science Foundation under Grant 61273142, Foundation for Six Talents by Jiangsu Province (2012-DZXX-045) and Priority Academic Program Development of Jiangsu Higher Education Institutions (PAPD)

\section{Conflict of interest}

The authors indicated no potential conflicts of interest. 


\section{References}

[1] S. Scalvini, E. Zanelli, M.A. Volterrani. Pilot study of nurse-led, home-based telecardiology for Patients with chronic heart failure. Journal of Telemedicine and Tele care, 2004, 10(2):113-117. https://doi.org/10.1258/135763304773391576

[2] W.T. Sung, K.Y. Chang. Health parameter monitoring via a novel wireless system. Applied Soft Computing, 2014, 22(5): 667-68. https://doi.org/10.1016/j.asoc.2014.04.036

[3] R. Haux, Medical informatics: past, present, future, International Journal of Medical Informatics, 2010, 79(9):599-610. https://doi.org/10.1016/j.ijmedinf.2010.06.003

[4] M. Paschou, E. Sakkopoulos, E. Sourla, A. Tsakalidis. Health Internet of Things: Metrics and methods for efficient data transfer. Simulation Modelling Practice and Theory, 2013, 34(5): 186-199. https://doi.org/10.1016/j.simpat.2012.08.002

[5] A. Belardinelli, G. Palagi, R. Bedini, A. Ripoli, V. Macellari, and D. Franchi. Advanced technology for personal biomedical signal logging and monitoring. In Proc. of the 20th Annual International Conference of the IEEE Engineering in Medicine and Biology Society, volume 3, pp.1295-1298, 1998. https://doi.org/10.1109/IEMBS.1998.747115

[6] E. Jovanov, T. Martin, and D. Raskovic. Issues in wearable computing for medical monitoring applications: A case study of a wearable ECG monitoring device. In Proc. of 4th International Symposium on Wearable Computers (ISWC), pp. 43-49, Oct. 2000.

[7] E. Jovanov, P. Gelabert, B. Wheelock, R. Adhami, and P. Smith. Real-time portable heart monitoring using low power DSP. In Proc. of International Conference on Signal Processing Applications and Technology ICSPAT, Oct. 2000.

[8] E. Jovanov, D. Raskovic, J. Price, A. Moore, J. Chapman, and A. Krishnamurthy. Patient monitoring using personal area networks of wireless intelligent sensors. Biomedical Sciences Instrumentation, 2001, 37(1):373-378.

[9] J. Bai, Y.H Zhang, D.L Shen, L.F Wen, et al. Portable ECG and blood pressure tele monitoring system. IEEE Engineering in Medicine and Biology, pp. 63-70, July/Aug.1999.

[10] P. Jelekainen, GSM-PKI solution enabling secure mobile communications, International Journal of Medical Informatics 2004, 73(3) 317-320. https://doi.org/10.1016/j.ijmedinf.20 03.12 .007

[11] S. Lei, W. Chen, L.H. Guo, et al. Study of algorithm for heart rate detection based on bipolar motion ECG. In Proc. of the 3rd International Conference on Measuring Technology and Mechatronics Automation, Piscataway.

[12] Smith D, Solem K, Laguna P, et al. Model-based detection of heart rate turbulence using mean shape Information. IEEE Transaction on Biomed Eng. 2010, 57(2):334-342. https://doi.org/10.1109/TBME.2009.2030669

[13] U. Anliker, J.A. Ward, P. Lukowicz, et al. AMON: a wearable multipara meter medical monitoring and alert system. IEEE Transaction on information technology in biomedicine, 2004, 8 (4):415-427. https://doi.org/10.1109/TITB.2004.837888

[14] N. Mi, L.A. Cavuoto, K. Benson, T. Smith-Jackson, M.A. Nussbaum. A heuristic checklist for an accessible smartphone interface design, Universal access in the information society 2013, 13(4):351-365. https://doi.org/10.1007/s10209-013-0321-4 


\section{Authors}

Haoran Li is the PH.D candidate of the School of Electrical \& Information Engineering in Jiangsu University. His major is control science. His current research interests include bio-information and fault diagnosis.

Tianhong Pan (corresponding author) is the professor of the School of Electrical \& Information Engineering in Jiangsu University. He has received Ph.D. degree in control theory and control engineering from Shanghai Jiao Tong University in 2007. His current research interests include multiple model approach and its application, machine learning, virtual metrology, predictive control and run-to-run control theory and practice, system identification (mobile: (86)15805298357, Fax: (86)51188790618, E-mail: thpan@ujs.edu.cn).

Article submitted 16 July 2017. Published as resubmitted by the authors 25 August 2017. 